\title{
ON THE FIXED-POINT THEORY FOR LOCAL $k$-PSEUDOCONTRACTIONS
}

\author{
CLAUDIO MORALES
}

\begin{abstract}
Two commonly used boundary conditions which imply existence of fixed points for local strong pseudocontractions in Banach spaces are compared, a previous fixed-point theorem for this class of mappings is improved, and an almost fixed-point result is obtained for local pseudocontractions.
\end{abstract}

Throughout this paper we suppose $X$ is a Banach space and $D$ an open subset of $X$. We use $B(x ; r)$ to denote the closed ball centered at $x \in X$ with radius $r>0$ and $\bar{D}$ and $\partial D$ to denote, respectively, the closure and boundary of $D$.

An operator $T: D \rightarrow X$ is said to be a local $k$-pseudocontraction $(k>0)$ (see [6]) if each point $x \in D$ has a neighborhood $N$ for which

$$
(\lambda-k)\|u-v\|<\|(\lambda I-T)(u)-(\lambda I-T)(v)\|, \quad u, v \in N, \lambda>k .
$$

For $k<1(k=1)$ such mappings are said to be local strong pseudocontractions (resp., local pseudocontractions). It is easy to verify that the condition (1) is implied by the assumption that $T$ is a local contraction with (uniform) Lipschitz constant $k<1(\|T(u)-T(v)\|<k\|u-v\|, u, v \in N)$, and in fact all of our results appear to be new even for this more restricted class of mappings.

Our main objective in this paper is to clarify the relationship between two conditions which have been prominently used in the recent development of fixed-point theory for the local and global strong pseudocontractions. These conditions are the standard Leray-Schauder condition (see, e.g., [2], [6]) which asserts that the mapping $T: \bar{D} \rightarrow X$ satisfies for some $z \in D$ :

$$
T(x)-z \neq \lambda(x-z) \text { for all } x \in \partial D \text { and } \lambda>1 \text {, }
$$

and the assumption (used in [1], [3], [4]) that the mapping $I-T$ not assume its infimum on $\partial D$. We show in fact, that for local strong pseudocontractions these conditions are in some sense equivalent. In the process we are able to sharpen Theorem 1 of [2] by showing that boundedness of the domain $D$ is not an essential assumption. This fact is a consequence of the implication (iii) $\Rightarrow$ (ii) in Theorem 1 below. We also obtain (Theorem 2) a localization of a result of [3].

Received by the editors August 24, 1979.

1980 Mathematics Subject Classification. Primary 47H10.

Key words and phrases. Local strong pseudocontraction, local pseudocontraction, fixed points. 
Theorem 1. Let $X$ be a Banach space, $D$ an open subset of $X$, and $T: \bar{D} \rightarrow X$ a continuous mapping which is a local strong pseudocontraction on $D$. Then the following are equivalent:

(i) $T$ has a fixed point in $D$.

(ii) There exists $z \in D$ such that $\|z-T z\|<\|x-T(x)\|$ for all $x \in \partial D$.

(iii) There exist an open set $G \subset D$ and $z \in G$ such that $T(x)-z \neq \lambda(x-z)$ for $x \in \partial G$ and $\lambda>1$.

The equivalence of (i) and (ii) is simply Theorem 3 of Kirk-Schöneberg [4]. Before proving the other implications we need some preliminary facts.

Lemma 1. Let $X$ be a Banach space, $D$ an open subset of $X$, and $T: \bar{D} \rightarrow X a$ continuous mapping which is a local $k$-pseudocontraction on $D$ (with $k<1$ ). Suppose that $T\left(x_{0}\right)=\lambda_{0} x_{0}$ for some $x_{0} \in D$ and $\lambda_{0}>1$, and suppose for $\sigma_{0}>0$, $B\left(x_{0} ; \sigma_{0}\right) \subset D$. Then:

(a) If $k<1$, for all $\lambda>1$ satisfying $\left|\lambda-\lambda_{0}\right|<\sigma_{0}(1-k) /\left\|x_{0}\right\|$, there exists a unique point $x_{\lambda} \in B\left(x_{0} ; \sigma_{0}\right)$ such that $T\left(x_{\lambda}\right)=\lambda x_{\lambda}$, and moreover this point satisfies

$$
\left\|x_{\lambda}-x_{0}\right\|<\left\|x_{\lambda}\right\|\left|\lambda-\lambda_{0}\right| /\left(\lambda_{0}-k\right) \text {. }
$$

(b) If $k=1$, for all $\lambda>1$ satisfying $\left|\lambda-\lambda_{0}\right|<\sigma_{0}\left(\lambda_{0}-1\right) /\left\|x_{0}\right\|$, there exists $a$ unique point $x_{\lambda} \in B\left(x_{0} ; \sigma_{0}\right)$ such that $T\left(x_{\lambda}\right)=\lambda x_{\lambda}$, and moreover this point satisfies

$$
\left\|x_{\lambda}-x_{0}\right\|<\left\|x_{\lambda}\right\|\left|\lambda-\lambda_{0}\right| /\left(\lambda_{0}-1\right) \text {. }
$$

Proof. (a) is Proposition 3 of [2] for $H=X$, and (b) is obtained by applying (a) to the mapping $\lambda_{0}^{-1} T$ (taking $k=\lambda_{0}^{-1}$ ).

LEMMA 2. Let $X$ be a Banach space, $D$ an open subset of $X$ and $T: \bar{D} \rightarrow X$ a continuous mapping which is a local $k$-pseudocontraction on $D$, with $k \in(0,1]$. For $H \subset D$, set $\mathcal{E}_{H}=\{\lambda>1: T(x)=\lambda x$ for some $x \in H\}$ and let $E=\{x \in D$ : $T(x)=\lambda x$ for some $\lambda>1\}$. Then

(i) the set $E$ is the union of disjoint nontrivial components, each of which is a continuous image of a subinterval of $[1, \infty)$.

In addition, if $F$ is any component of $E$, then

(ii) the function $h: \mathcal{E}_{F} \rightarrow \mathbf{R}$ defined by $h(\lambda)=\left\|x_{\lambda}-T\left(x_{\lambda}\right)\right\|$, where $x_{\lambda} \in F$ and $T\left(x_{\lambda}\right)=\lambda x_{\lambda}$, is nondecreasing;

(iii) if $k<1<\lambda_{0}$ and $\lambda_{0} \in \mathcal{E}_{F}$, then the set $S=\{x \in F$ : $T(x)=\lambda x$ for some $\left.\lambda \in \mathcal{E}_{F} \cap\left(1, \lambda_{0}\right]\right\}$ is bounded; and

(iv) if $T\left(x_{\lambda_{n}}\right)=\lambda_{n} x_{\lambda_{n}}$ with $\lambda_{n} \rightarrow \lambda>k\left(\lambda_{n}>\lambda\right.$ and $\left.k<1\right)$ and $\left\{x_{\lambda_{n}}\right\} \subset F$, then $\left\{x_{\lambda_{n}}\right\}$ is a Cauchy sequence.

Also:

(v) If $0 \in D$, then there exists $r>0$ such that $(r, \infty) \subset \mathcal{E}_{D}$.

Proof. (i) is an immediate consequence of Lemma 1.

(ii) Let $\lambda \in \mathcal{E}_{F}$ and $x_{\lambda} \in F$ with $T\left(x_{\lambda}\right)=\lambda x_{\lambda}$. Then, by assumption, there exists $\sigma>0$ such that $T$ is a $k$-pseudocontraction on $B\left(x_{\lambda} ; \sigma\right) \subset D$. Suppose $T\left(x_{\mu}\right)=\mu x_{\mu}$ where $x_{\mu} \in B\left(x_{\lambda} ; \sigma\right) \cap F$ and $1<\mu<\lambda$. Then by (1) we have 


$$
\begin{aligned}
(\mu-k)\left\|x_{\lambda}-x_{\mu}\right\| & <\left\|\mu x_{\lambda}-T\left(x_{\lambda}\right)-\left(\mu x_{\mu}-T\left(x_{\mu}\right)\right)\right\| \\
& =(\lambda-\mu)\left\|x_{\lambda}\right\|,
\end{aligned}
$$

which yields

$$
\begin{aligned}
\left\|x_{\mu}-T\left(x_{\mu}\right)\right\| & =(\mu-1)\left\|x_{\mu}\right\|<(\mu-1)\left[\left\|x_{\mu}-x_{\lambda}\right\|+\left\|x_{\lambda}\right\|\right] \\
& \leqslant(\mu-1)[(\lambda-\mu) /(\mu-k)+1]\left\|x_{\lambda}\right\| \\
& \leqslant(\lambda-1)\left\|x_{\lambda}\right\|=\left\|x_{\lambda}-T\left(x_{\lambda}\right)\right\| .
\end{aligned}
$$

(iii) Suppose $x_{\lambda_{0}} \in F$ with $T\left(x_{\lambda_{0}}\right)=\lambda_{0} x_{\lambda_{0}}$ and select $\mu, x_{\mu}$ as in (ii). Then by (2) we have

$$
\begin{aligned}
(\mu-k)\left\|x_{\mu}\right\| & <(\mu-k)\left[\left\|x_{\lambda_{0}}-x_{\mu}\right\|+\left\|x_{\lambda_{0}}\right\|\right] \\
& \leqslant\left(\lambda_{0}-k\right)\left\|x_{\lambda_{0}}\right\|,
\end{aligned}
$$

and it follows that $\left\|x_{\mu}\right\|<\left\|x_{\lambda_{0}}\right\|\left|\lambda_{0}-k\right| /(1-k)$ for all $x_{\mu} \in S$.

(iv) We follow the proof of Theorem 1 of [2]. If $\lambda_{n}<\lambda_{m}$, then by Lemma 1 the segment $\left[\lambda_{n}, \lambda_{m}\right]$ can be covered by a finite number of overlapping subintervals $\left\{I_{i}\right\}_{i=1}^{n}$ which have the property that for each $i$ and $\lambda, \mu \in I_{i}$, the eigenvectors $x_{\lambda}, x_{\mu}$ corresponding to $\lambda$ and $\mu$ satisfy

$$
\left\|x_{\lambda}-x_{\mu}\right\| \leqslant M|\lambda-\mu| /(1-k),
$$

where $M=\sup \left\{\left\|x_{\lambda}\right\|: x_{\lambda} \in F, 1<\lambda<\lambda_{0}\right\}$ with $\lambda_{0}=\sup \left\{\lambda_{n}\right\}$. (By (iii), $M<\infty$.)

We may now select $\mu_{i} \in I_{i} \cap I_{i+1}$ such that $\lambda_{n}=\mu_{0}<\mu_{1}<\cdots<\mu_{r+1}=\lambda_{m}$. Then by (3),

$$
\left\|x_{\mu_{i}}-x_{\mu_{i+1}}\right\| \leqslant M\left|\mu_{i}-\mu_{i+1}\right| /(1-k), \quad i=0,1, \ldots, r,
$$

and thus

$$
\left\|x_{\lambda_{h}}-x_{\lambda_{m}}\right\| \leqslant \sum_{i=0}^{r}\left\|x_{\mu_{i}}-x_{\mu_{i+1}}\right\| \leqslant M \sum_{i=0}^{r}\left|\mu_{i}-\mu_{i+1}\right| /(1-k) .
$$

Therefore, $\left\{x_{\lambda_{n}}\right\}$ is a Cauchy sequence.

(v) By the continuity of $T$ at 0 , there exists $\sigma \in(0,1)$ and a ball $B$ centered at the origin such that (1) holds for all $u, v \in B$ while $t T(B) \subset B$ for all $t<\sigma$. Since (1) implies for $u, v \in B:\langle u-v, T(u)-T(v)\rangle_{-}\left\langle k\|u-v\|^{2}\right.$ (in the notation of Martin [5]), it follows from Theorem 6 of [5] that $\left(\sigma^{-1}, \infty\right) \subset \mathcal{E}_{D}$.

Lemma 3. Let $X$ be a Banach space, $D$ an open subset of $X$ and $T: D \rightarrow X a$ continuous local pseudocontraction. Then the set $\mathcal{E}_{D}=\{\lambda>1: T(x)=\lambda x$ for some $x \in D\}$ is open in $(1, \infty)$.

Proof. This is an immediate consequence of Lemma 1(b).

We now complete the proof of Theorem 1.

Proof of Theorem 1. (ii) $\Rightarrow$ (iii). By replacing $T(x)$ with $T(x+z)-z$ one may take $z=0$ in (ii) (and thus by assumption $0 \in D$ ). Define $E$ as in Lemma 2 and let $F_{0}$ be the component of $E$ for which $0 \in \bar{F}$. Let $B$ be a ball centered at 0 such that $T$ restricted to $B$ is a strong pseudocontraction, and let $x \in B \cap F_{0}$ with $T(x)=$ $\lambda x$. Then by (1)

$$
(\lambda-1)\|x\| \leqslant\|\lambda x-T(x)+T(0)\|=\|T(0)\|,
$$


yielding $\|x-T(x)\|<\|T(0)\|$. Therefore, Lemma 2(ii) and Lemma 1 imply that $\|x-T(x)\|<\|T(0)\|$ for all $x \in F_{0}$, proving $F_{0} \subset D$. Since $F_{0}$ is a component of $E$, there exists an open set $U$ in $X$ such that $U \cap E=F_{0}$. Choosing $G=D \cap U$ completes the proof of (iii).

(iii) $\Rightarrow$ (i). Without loss of generality, we may assume $z=0$ in (iii). As in the proof of Lemma $2(\mathrm{v})$, there exists $\sigma \in(0,1)$ such that $t T(B) \subset B$ for some ball $B$ centered at the origin and for all $t<\sigma$. Hence $\lambda \in \mathcal{E}_{F_{0}}$ and $x_{\lambda} \in B$ for all $\lambda>\sigma^{-1}$; thus by Lemma 2(iii) we conclude that $F_{0}$ (defined as above) is bounded. Therefore, there is no loss in generality in assuming $G$ bounded, and existence of a fixed point for $T$ in $D$ follows from Corollary 1 of Kirk-Morales [2].

The next theorem represents a localized version of the first part of Theorem 1 of [3].

TheOREM 2. Let $X$ be a Banach space, $D$ a bounded open subset of $X$, and $T$ : $\bar{D} \rightarrow X$ a continuous mapping which is a local pseudocontraction on $D$. Suppose there exists $z \in D$ such that

$$
\|z-T(z)\|<\|x-T(x)\| \text { for all } x \in \partial D .
$$

Then $\inf \{\|x-T(x)\|: x \in \bar{D}\}=0$.

Proof. As before, by replacing $T(x)$ with $T(x+z)-z$ and $D$ by $D-z$, one may take $z=0$ in (4). Since $T$ is continuous and $0 \in D, \mathcal{E}_{D} \neq \varnothing$ (by Lemma 2(v)). Let $\lambda_{0}=\inf \mathcal{E}_{F_{0}}$ where $F_{0}$ is the component of $E$ containing 0 in its closure. Suppose first that $\lambda_{0}>1$. Then by Lemma 2(iv), $\lambda_{0} \in \mathcal{E}_{\bar{D}}$, i.e., $T\left(x_{0}\right)=\lambda_{0} x_{0}$ for some $x_{0} \in \bar{D}$. If $x_{0} \in D$, Lemma 3 implies that $\lambda \in \mathcal{E}_{F_{0}}$ for some $\lambda<\lambda_{0}$, which contradicts the minimality of $\lambda_{0}$. It follows in this case that $\lambda_{0}=1$, and thus $\inf \{\|x-T(x)\|: x \in \bar{D}\}=0$. To complete the proof, observe that as in the proof of Theorem 1, $\|x-T(x)\|<\|T(0)\|$ for all $x \in F_{0}$. Thus, by continuity of $T$ on $\bar{D}$, $\left\|x_{0}-T\left(x_{0}\right)\right\| \leqslant\|T(0)\|$, and it follows from (4) that $x_{0} \notin \partial D$.

It is shown in [1] that for uniformly convex $X$ the assumptions of Theorem 2 guarantee existence of a fixed point for $T$.

\section{REFERENCES}

1. W. A. Kirk, A fixed point for local pseudo-contractions in uniformly convex spaces, Manuscripta Math. 30 (1979), 89-102.

2. W. A. Kirk and C. Morales, Fixed point theorems for local strong pseudo-contractions, nonlinear analysis, Theory, Methods, and Applications 4 (1980), 363-368.

3. W. A. Kirk and R. Schöneberg, Some results on pseudo-contractive mappings, Pacific J. Math. 71 (1977), 89-100.

4. Mapping theorems for local expansions in metric and Banach spaces, J. Math. Anal. Appl. 72 (1979), 114-121.

5. R. H. Martin, Jr., Differential equations on closed subsets of a Banach space, Trans. Amer. Math. Soc. 179 (1973), 399-414.

6. C. Morales, Pseudo-contractive mappings and the Leray-Schauder boundary condition, Comment. Math. Univ. Carolinae 20 (1979), 745-756.

Department of Mathematics, Universtty of Iowa, Iowa CtTy, Iowa 52242 